\title{
TURBULENCE DRIVEN BY OUTFLOW-BLOWN CAVITIES IN THE MOLECULAR CLOUD OF NGC 1333
}

\author{
Alice C. Quillen, Stephen L. Thorndike, Andy Cunningham, Adam Frank, \\ Robert A. Gutermuth, Eric G. Blackman, and Judith L. Pipher \\ Department of Physics and Astronomy, University of Rochester, Rochester, NY 14627; aquillen@pas.rochester.edu \\ AND \\ NAOMI RIDGE \\ Harvard-Smithsonian Center for Astrophysics, 60 Garden Street, Cambridge, MA 02138 \\ Received 2005 March 7; accepted 2005 June 23
}

\begin{abstract}
Outflows from young stellar objects (YSOs) have been identified as a possible source of turbulence in molecular clouds. To investigate the relationship between outflows, cloud dynamics, and turbulence, we compare the kinematics of the molecular gas associated with NGC 1333 , traced in ${ }^{13} \mathrm{CO}(1-0)$, with the distribution of YSOs within. We find a velocity dispersion of $\sim 1-1.6 \mathrm{~km} \mathrm{~s}^{-1}$ in ${ }^{13} \mathrm{CO}$ that does not significantly vary across the cloud and is uncorrelated with the number of nearby young stellar outflows identified from optical and submillimeter observations. However, from velocity channel maps we identify about 20 depressions in the ${ }^{13} \mathrm{CO}$ intensity of scales $\gtrsim 0.1-0.2 \mathrm{pc}$ and velocity widths $1-3 \mathrm{~km} \mathrm{~s}^{-1}$. The depressions exhibit limb-brightened rims in both individual velocity channel maps and position-velocity diagrams, suggesting that they are slowly expanding cavities. We interpret these depressions to be remnants of past YSO outflow activity: if these cavities are presently empty, they would fill in on timescales of $\sim 10^{6} \mathrm{yr}$. This can exceed the lifetime of a YSO outflow phase or the transit time of the central star through the cavity, explaining the absence of any clear correlation between the cavities and YSO outflows. We find that the momentum and energy deposition associated with the expansion of the cavities is sufficient to power the turbulence in the cloud. In this way we conclude that the cavities are an important intermediate step between the conversion of YSO outflow energy and momentum into cloud turbulent motions.
\end{abstract}

Subject headings: ISM: bubbles — ISM: clouds — ISM: individual (NGC 1333) — ISM: jets and outflows ISM: kinematics and dynamics - ISM: molecules

Online material: color figures

\section{INTRODUCTION}

Energetic outflows exert a strong effect on their parent molecular clouds (for a recent review see Reipurth \& Bally 2001). Measurements of the total kinetic energy present in young stellar outflows imply that outflows and winds associated with young stellar objects (YSOs) contain sufficient kinetic energy to excite a significant fraction of the supersonic turbulence present in molecular clouds and/or unbind and disperse the cloud (Bally et al. 1996a; Reipurth \& Bally 2001; Knee \& Sandell 2000; Warin et al. 1996).

The molecular cloud associated with the reflection nebula NGC 1333 provides a good setting to study the relation between young stellar outflows and their influence on parent clouds because it is an active site of low- and intermediate-mass star formation. NGC 1333 contains two young star clusters identified in near-infrared studies (Aspin et al. 1994; Lada et al. 1996), many Herbig-Haro (HH) objects, and associated molecular outflows (e.g., Bally et al. 1996b; Aspin 2003; Knee \& Sandell 2000), as well as younger, embedded $I R A S$ sources (Sandell \& Knee 2001; Jennings et al. 1987; Rodriguez et al. 1999).

Previous studies have suggested that the outflows present in NGC 1333 could have perturbed the associated molecular cloud. Based on his study of the star clusters, Aspin (2003) suggested that the large number of active molecular outflows in the southern region of NGC 1333 could provide a mechanism for exciting cloud turbulence and causing subsequent star formation. Based on a study of the cloud in ${ }^{12} \mathrm{CO}(3-2)$, Knee \& Sandell (2000) found that molecular outflows observed in the cloud could provide enough kinetic energy to accelerate the entire cloud by a few kilometers per second. Dust ridges and shells seen in submillimeter continuum can be associated with outflows, implying that the cloud itself has been considerably modified and, perhaps, disrupted by the outflows (Sandell \& Knee 2001; Warin et al. 1996; Lefloch et al. 1998; Bally et al. 1996b).

Many authors have argued that outflows could stimulate star formation or be responsible for turbulence in molecular clouds. These arguments usually rely on estimates of the energy present in outflows compared to that associated with turbulence in the clouds (Reipurth \& Bally 2001). Studies of individual objects, however, express a more complicated picture. In particular, the explicit nature of the coupling between cloud material and outflows could be better explored. Molecular outflows are identified through millimeter radio emission from molecules such as $\mathrm{CO}$ extending beyond the more collimated emission seen in HH objects in the visible (e.g., Bence et al. 1996; Yu et al. 1999). In giant outflows, $\mathrm{CO}$ emission associated with the outflow can extend to a few arcminutes from the central source (Chernin \& Masson 1995; Cernicharo \& Reipurth 1996). Unified models suggest that it is the narrow bipolar jet with, perhaps, a wideangle wind component that entrains molecular material, which is then seen as the CO molecular outflow (Raga \& Cabrit 1993; Chernin et al. 1994; Delamarter et al. 2000; Lee et al. 2002; Arce \& Sargent 2004; Gardiner et al. 2003). We note that high angular resolution observational studies have revealed evidence for wideangle winds in addition to collimated jets (e.g., Arce \& Goodman 2002b). 
In ${ }^{12} \mathrm{CO}$ lines such as the $J=1-0$ transition the optical depth increases rapidly near the velocity centroid of a molecular cloud. However, the optical depth decreases with increasing velocity offset and so ${ }^{12} \mathrm{CO}$ can be used to trace lower density material associated with outflows (e.g., the survey of NGC 1333 by Knee $\&$ Sandell 2000). However, to trace the structure at higher densities, a more optically thin tracer such as ${ }^{13} \mathrm{CO}$ or $\mathrm{C}^{18} \mathrm{O}$ is needed. Ridge et al. (2003) have carried out a survey of nearby molecular clouds in these two emission lines, providing a database with which to investigate the mechanism and timescale for the dissipation of gas around individual stars and clusters. NGC 1333 has been previously surveyed in the same molecules by Warin et al. (1996). The overall structure of the warm gas in the cloud is expected to be well traced by the $\mathrm{C}^{18} \mathrm{O}$ emission, with depletion or freezeout of $\mathrm{CO}$ only occurring in small dense pockets (Bergin et al. 2002).

Outflows and winds associated with YSOs produce highvelocity gas but also can evacuate regions in their host molecular cloud. Cavities associated with outflows have been seen previously, particularly in low-opacity tracers such as ${ }^{13} \mathrm{CO}$ (e.g., Warin et al. 1996; Arce \& Goodman 2002a; Lefloch et al. 1998; Welch et al. 2000). In the Circinus cloud, Bally et al. (1999) described dusty filaments confining $1 \mathrm{pc}$ sized cavities that were also seen in their ${ }^{13} \mathrm{CO}$ and $\mathrm{C}^{18} \mathrm{O}$ maps. They suggested that these cavities were fossil remnants of previous outflows. Snell et al. (1980) described a $15 \mathrm{~km} \mathrm{~s}^{-1}$ velocity, parsec-sized double-lobed cavity associated with L1551. Reipurth et al. (1998) described an evacuated parsec-long chimney associated with $\mathrm{HH} 310$. With higher resolution CO interferometry, Lee et al. (2002) described complex outflow morphology such as shell-like and multipolar structures, multiple cavities, and asymmetric lobes. Arce \& Sargent (2004) showed that the outflow of L1228 had evacuated a conical-shaped cavity and had morphology and kinematics consistent with entrainment of gas by both collimated and wide-angle outflow components. Welch et al. (2000) showed that a 0.05 pc expanding shell in Taurus could have been caused by outflows driven by the binary XZ Taurus. In the NGC 1333 cloud, Warin et al. (1996) suggest that the SVS 13, IRAS 2, and IRAS 4 outflows have created the cavity that lies between SVS 13 and HH 12. However, Knee \& Sandell (2000) found that the molecular outflow associated with IRAS 4 did not overlap with this cavity.

In a few cases, theoretical models for expanding spherical cavities, i.e., wind-blown bubbles, have been applied to cavities opened by outflows. Koo \& McKee (1992a) applied their windblown bubble model to the HH 7-11 region, located in the NGC 1333 cloud. In the context of a slow wind, Koo \& McKee (1992a) suggested that cavities opened in molecular clouds by outflows were consistent with their estimated mechanical luminosities. Raga et al. (2004) modeled the limb-brightened cavity associated with HH 46/47 (Noriega-Crespo et al. 2004) as a bow shock driven by a perfectly collimated jet.

Most observational studies have suggested that existing $\mathrm{HH}$ objects and outflows could be responsible for creating cavities (e.g., Knee \& Sandell 2000; Koo \& McKee 1992a; Warin et al. 1996; Lefloch et al. 1998; Lee et al. 2002; Arce \& Goodman 2002a; Noriega-Crespo et al. 2004; Raga et al. 2004) and have identified the most prominent outflows evident in the winds of the $\mathrm{CO}$ channel maps or seen as $\mathrm{HH}$ objects with the most prominent cavities. Since the duration of the strong outflow period of a protostar may be short compared with the life of a young cluster, the history of the coupling between the outflow and molecular cloud must be considered. Indeed, some studies have also suggested that cavities in molecular clouds could be tracers of previously active outflows (e.g., Bally et al. 1999). In the vicinity of

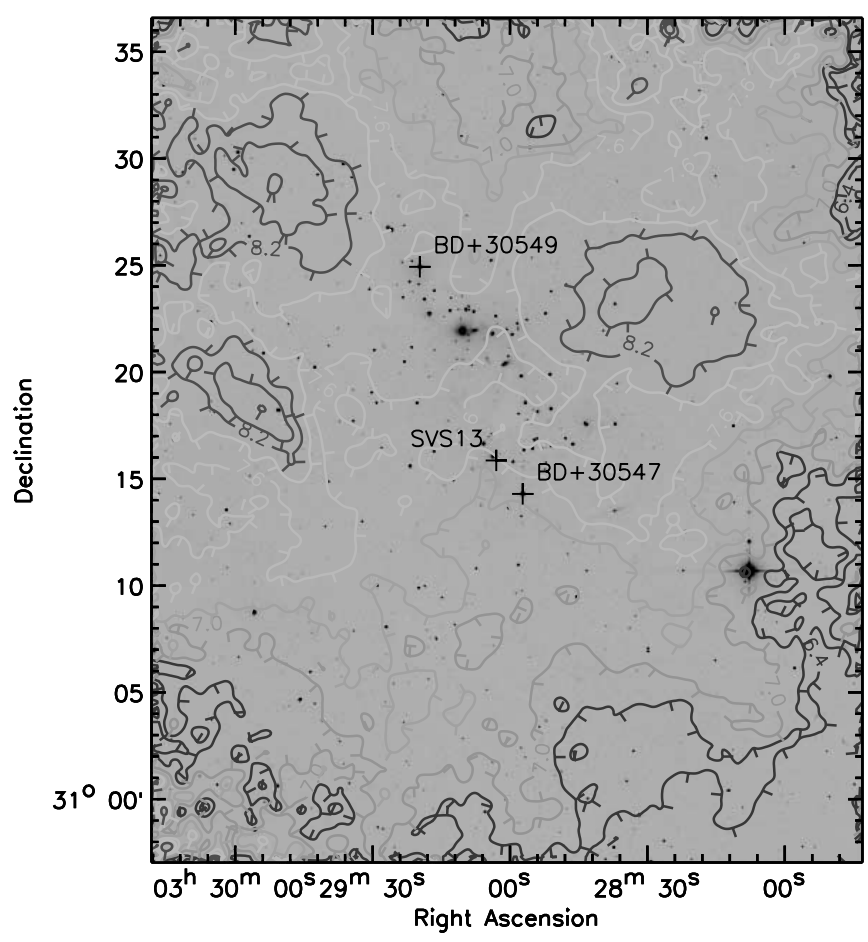

FIG. 1.-Mean velocity of the ${ }^{13} \mathrm{CO}(1-0)$ velocity cube contours (see Ridge et al. 2003) overlayed on the $K_{s}$-band 2 MASS image shown as gray scale. Contour spacing is $0.3 \mathrm{~km} \mathrm{~s}^{-1}$ and given with respect to the local standard of rest. The bright region just to the left and below the center of the frame contains the star SVS 13, which has been used as a reference point by previous works (e.g., Knee $\&$ Sandell 2000). Also shown for reference are the B stars BD +30547 and $\mathrm{BD}+30549$. BD +30549 illuminates the reflection nebula. The region south of SVS 13 is moving at a higher mean velocity (by about $0.5 \mathrm{~km} \mathrm{~s}^{-1}$ ) than the northern region. The angular resolution of the ${ }^{13} \mathrm{CO}$ data is $47^{\prime \prime}$. Coordinates are given with respect to epoch J2000.0. [See the electronic edition of the Journal for a color version of this figure.]

SVS 13 in NGC 1333, both possibilities have been suggested (Warin et al. 1996; Lefloch et al. 1998). Warin et al. (1996) suggested that the star cluster north of SVS 13 was associated with a cavity, whereas Lefloch et al. (1998) identified two cavities south of SVS 13 and associated one them with a cone-shaped outflow emanating from the infrared source SVS 13.

To investigate the coupling between outflows and the dynamics of molecular clouds, we compare the spatial distribution of outflows in NGC 1333 with the properties of its molecular cloud. Specifically, we compare the lower optical depth ${ }^{13} \mathrm{CO}$ millimeter velocity cubes obtained by Ridge et al. (2003) with near-infrared sources and the locations of outflows identified by previous surveys. In addition, we compare structures seen in the molecular gas distribution with the locations of YSOs.

We adopt a distance to NGC 1333 of 212 pc based on the distance estimated to the star $\mathrm{BD}+30549$ that illuminates the nebula (Cernis 1990). This distance is nearer than the estimate $318 \mathrm{pc}$ that is based on the Hipparcos parallactic measurements of the Perseus OB2 association (de Zeeuw et al. 1999). At the distance of $212 \mathrm{pc}, 1^{\prime}$ corresponds to $0.062 \mathrm{pc}$.

\section{MEAN VELOCITY FIELD AND VELOCITY DISPERSION OF THE MOLECULAR GAS}

If outflows associated with YSOs are responsible for turbulence excited in the cloud, then we might expect regions that contain large numbers of outflows to reside in a more turbulent molecular medium. In this section we compare the integrated properties of the molecular gas, as traced in ${ }^{13} \mathrm{CO}$ by Ridge et al. 


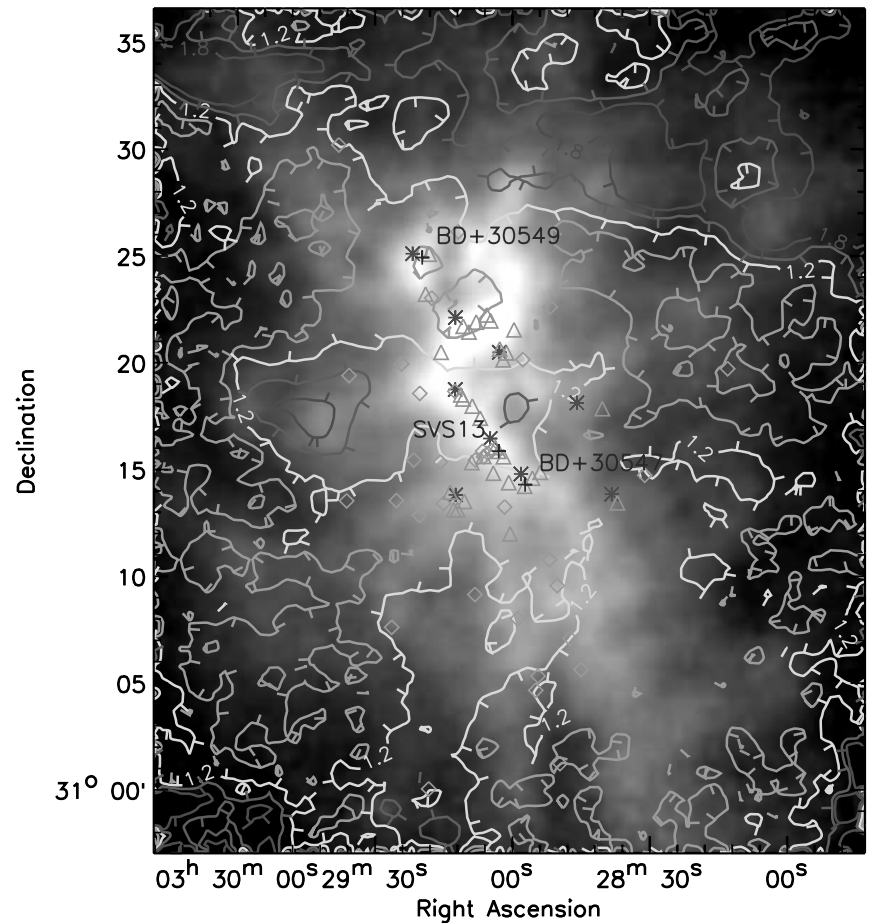

FIG. 2.-Velocity dispersion (rms) about the mean from the ${ }^{13} \mathrm{CO}(1-0)$ velocity cube overlayed on the integrated ${ }^{13} \mathrm{CO}$ intensity (gray scale). Contour spacing is $0.3\left(\mathrm{~km} \mathrm{~s}^{-1}\right)$. Much of the structure in the velocity dispersion map is due to the low signal-to-noise ratio at the cloud outer edges. While the cloud shows significant variations in the mean velocity, little variation in the velocity dispersion is seen across the cloud. For comparison, IRAS sources from Jennings et al. (1987) are shown by stars. HH objects from Reipurth (1999) are shown by diamonds. Compact submillimeter sources from Knee \& Sandell (2000) are shown by triangles. The B stars BD +30 547 and BD +30 549 and infrared source SVS 13 are shown by crosses. Two depressions in the molecular gas distribution are seen, one just to the northwest of SVS 13 and the other $7^{\prime}$ north and slightly east of SVS 13. These depressions are not coincident with bright infrared sources, $\mathrm{HH}$ objects, or early-type stars. [See the electronic edition of the Journal for a color version of this figure.]

(2003), with the distribution of previously identified outflow sources.

Of the NGC 1333 velocity cubes presented and discussed by Ridge et al. (2003), the highest signal-to-noise ratio data set for NGC 1333 is the ${ }^{13} \mathrm{CO}(1-0)$ data cube obtained at the Five College Radio Astronomy Observatory (FCRAO). Because of its higher signal-to-noise ratio, we primarily use this velocity set for our comparison. The angular resolution of these data is $47^{\prime \prime}$ and the spectral resolution corresponds to a velocity spacing between each channel of $0.133 \mathrm{~km} \mathrm{~s}^{-1}$. For NGC 1333, $47^{\prime \prime}$ corresponds to $\sim 0.05 \mathrm{pc}$.

In Figure 1 we show the mean velocity measured from the ${ }^{13} \mathrm{CO}(1-0)$ data cube as contours, overlaid on the Two Micron All Sky Survey (2MASS) $K_{s}$-band image shown as gray scale. In Figure 2 we show the velocity dispersion measured from the ${ }^{13} \mathrm{CO}(1-0)$ data cube, overlaid on the integrated intensity of the velocity cube. The mean velocity, $\langle v\rangle$, is the intensity-weighted mean velocity at each position of the map measured from the entire velocity cube and is computed as $\langle v\rangle=\sum_{i} v_{i} I_{i} / \sum_{i} I_{i}$, where $I_{i}$ and $v_{i}$ are the velocity and intensities of the individual pixels, respectively. The velocity dispersion, $\sigma$, is the square root of the intensity-weighted square of the velocity subtracted by the mean; $\sigma^{2}=\sum\left(v_{i}-\langle v\rangle\right)^{2} I_{i} / \sum_{i} I_{i}$.

We might expect an increase in the velocity dispersion of the molecular cloud in regions that contain many outflow sources or many embedded young objects. However, from inspection of

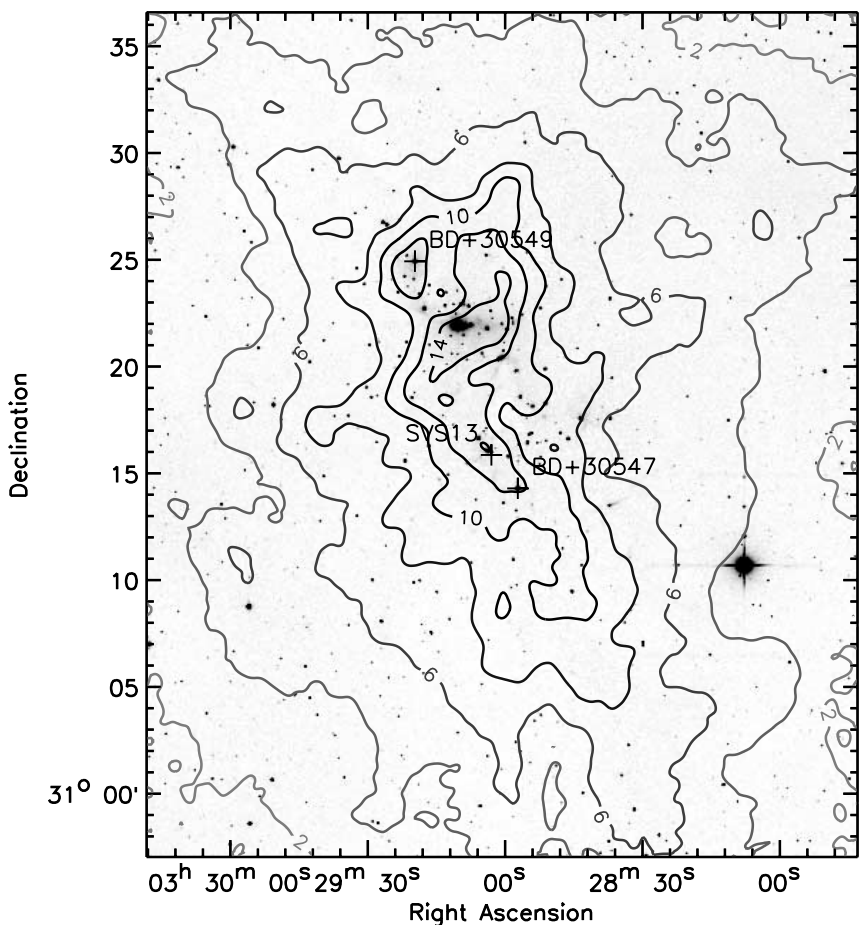

FIG. 3.- Integrated ${ }^{13} \mathrm{CO}$ intensity (contours) overlayed on top of the $K_{s}$-band 2MASS image ( gray scale). Contours are shown $2 \mathrm{~K} \mathrm{~km} \mathrm{~s}^{-1}$ apart with the lowest and highest contours at 2 and $14 \mathrm{~K} \mathrm{~km} \mathrm{~s}^{-1}$. The 2MASS image shows $\mathrm{T}$ Tauri cluster stars and background stars.

Figure 2 we find that the velocity dispersion as measured from ${ }^{13} \mathrm{CO}$ does not significantly vary across the molecular cloud. The velocity dispersion ranges between 1.0 and $1.6 \mathrm{~km} \mathrm{~s}^{-1}$ across the cloud. This velocity range is well within the blueshifted wings seen in ${ }^{12} \mathrm{CO}$ that are $10 \mathrm{~km} \mathrm{~s}^{-1}$ below the cloud velocity mean from outflows associated with the most prominent $\mathrm{HH}$ objects (Knee \& Sandell 2000).

Hydrostatic equilibrium models for clouds predict relations between velocity dispersion, density, and radius (e.g., Myers \& Fuller 1992). However, Figure 2 shows no strong relation between integrated intensity and velocity dispersion, as would be expected from hydrostatic cloud models. There is little significant structure in the constant dispersion contours, although the dispersion is somewhat higher near the lower ${ }^{13} \mathrm{CO}$ intensity region to the north of SVS 13 . The somewhat higher velocity dispersion in this depression or cavity might be explained with a scenario in which a cavity is evacuated by winds driven from sources within the cavity. Two star clusters have been identified in the near-infrared imaging by Aspin et al. (1994). One cluster is located in the vicinity of this cavity, and another is located near the depression in the integrated ${ }^{13} \mathrm{CO}$ about $7^{\prime}$ to the north of SVS 13. These clusters can be seen in the $K$-band stellar distribution in Figure 3. The outflow region associated with SVS 13 (the HH 7-11 region) does not have a larger velocity dispersion than other regions of the cloud. Likewise, the $\mathrm{HH}$ 4, HH 2, and HH 12 regions associated with outflows seen in ${ }^{12} \mathrm{CO}$ by Knee \& Sandell (2000) also do not stand out in the velocity dispersion maps. Significant structure correlated with positions of known outflows in the cloud random motions as traced by the velocity dispersion is not observed in the lower opacity tracer ${ }^{13} \mathrm{CO}$.

Outflows previously identified in ${ }^{12} \mathrm{CO}$ as high-velocity wings (with blueshifted and redshifted emission greater than a few kilometers per second from the cloud mean) by Knee \& Sandell 


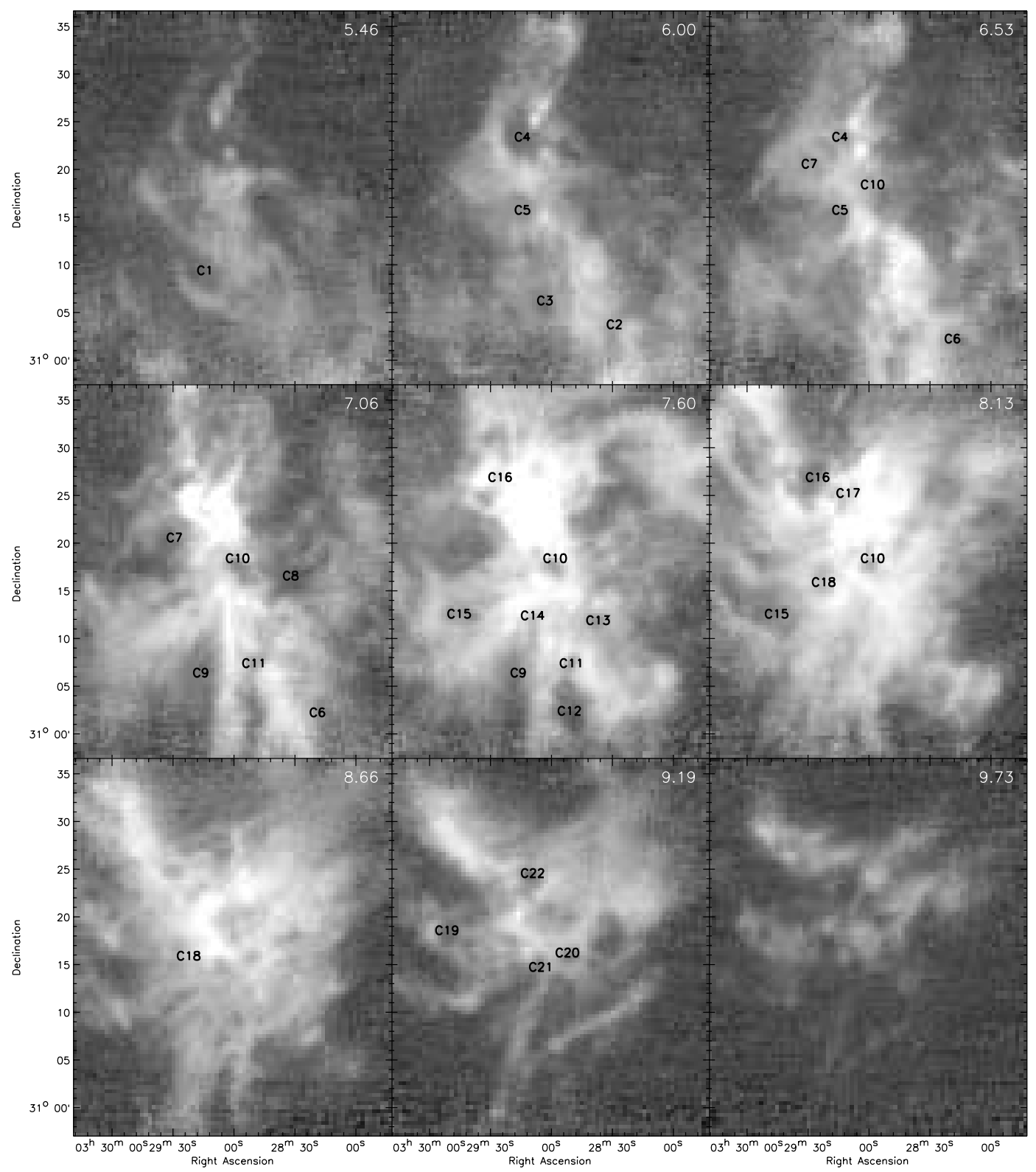

FIG. 4.-Channel maps for a subsample of the velocity cube. The velocity spacing between the channel maps displayed is four channels or $0.53 \mathrm{~km} \mathrm{~s}{ }^{-1}$. Each channel has a velocity width of $0.133 \mathrm{~km} \mathrm{~s}^{-1}$. The velocity of the channel shown in each panel is shown in the upper right corner of the image. Multiple shells and cavities are evident in the individual channel maps. Depressions in the intensity identified in both position-velocity diagrams and individual channel maps are labeled here on the channel maps. The properties of these cavities are listed in Table 1.

(2000) are not evident in the high-velocity wings in the ${ }^{13} \mathrm{CO}$ or $\mathrm{C}^{18} \mathrm{O}$ data of Ridge et al. (2003). At velocities below $5 \mathrm{~km} \mathrm{~s}^{-1}$ and above $10 \mathrm{~km} \mathrm{~s}^{-1}$ little emission is detected in the individual ${ }^{13} \mathrm{CO}$ channel maps (see Fig. 4). Knee \& Sandell (2000) found outflows associated with HH 6, HH 12, HH 7-11, IRAS 2, and IRAS 4; their CO maps did not extend all the way to IRAS 1. Surveys in ${ }^{12} \mathrm{CO}$ detect outflows, whereas those in ${ }^{13} \mathrm{CO}$ often do not (e.g., Warin et al. 1996). This is most likely because the ${ }^{12} \mathrm{CO}$ emission is more sensitive to optically thin and low-density regions but less capable of penetrating denser, higher opacity regions (e.g., Arce \& Sargent 2004). A deeper, more sensitive velocity cube in ${ }^{13} \mathrm{CO}$ might reveal additional structure near the individual outflows, particularly at velocities more than $3 \mathrm{~km} \mathrm{~s}^{-1}$ above or below the mean cloud velocity. Outflows traced in the high-velocity wings of molecules such as ${ }^{12} \mathrm{CO}$ are associated with a low-density medium that is faint and so difficult to detect in ${ }^{13} \mathrm{CO}$.
Because we see little high-velocity gas in ${ }^{13} \mathrm{CO}$ and no large spatial variations in the velocity dispersion, we find little evidence for turbulence in the denser regions of the molecular cloud that has been directly induced by the high concentration of high-velocity outflows discovered at other wavelengths. We conclude that the bulk of the cloud, which we expect is traced in ${ }^{13} \mathrm{CO}$, does not contain much high-velocity gas associated with active outflows.

In contrast to the velocity dispersion map, there is quite a bit of structure in the mean velocity field shown in Figure 1. The mean velocity field exhibits a gradient with velocity increasing from the south to the north of the cloud by about $1 \mathrm{~km} \mathrm{~s}^{-1}$. There is also a jump in velocity of $\sim 0.3 \mathrm{~km} \mathrm{~s}^{-1}$ separating the southern region in NGC 1333S from the rest of the cluster.

\section{CAVITIES}

In the previous section we found that the integrated properties of the ${ }^{13} \mathrm{CO}$ velocity cube were not strongly influenced by 
outflows. In this section we inspect individual channel maps and position-velocity diagrams from the ${ }^{13} \mathrm{CO}$ velocity cube to better investigate the possibility that outflows have affected the structure of the molecular cloud.

Instead of high-velocity gas associated with outflows, we see shells and cavities or depressions in the gas distribution in individual channel maps (see Fig. 4). Cavities in the ${ }^{13} \mathrm{CO}$ integrated intensity have been described by previous studies (e.g., Warin et al. 1996; Lefloch et al. 1998). Because these depressions in intensity are seen in more than one neighboring velocity channel, they are likely to be real and not a result of noise in the data. Different cavities appear at different velocities, suggesting that they are often not directly related to one another. Some cavities can disappear at velocities higher or lower than the central value where the depression is most prominent, although CO emission can remain strong. This suggests that the cavities can lie within the cloud and are not exclusively a result offilamentary structure near the cloud edges. Previous studies comparing maps of line emission in $\mathrm{C}^{13} \mathrm{O},{ }^{13} \mathrm{CO}, \mathrm{NH}_{3}$, and $\mathrm{CS}$ have found that depressions in the line intensity in ${ }^{13} \mathrm{CO}$ and $\mathrm{C}^{18} \mathrm{O}$ are primarily due to lower gas density rather than depletion of $\mathrm{CO}$, temperature variations, or high opacity (Warin et al. 1996; Lefloch et al. 1998). From inspection of the channel maps, we find that the most prominent cavities are also evident in the lower opacity and lower signal-to-noise ratio $\mathrm{C}^{18} \mathrm{O}$ velocity cube of $\mathrm{NGC} 1333$ (for additional discussion on morphology in $\mathrm{C}^{18} \mathrm{O}$ compared to that seen in ${ }^{13} \mathrm{CO}$ see $\S 3.1$ ).

The velocity gradient across the NGC 1333 molecular cloud is fairly smooth or shallow (Fig. 1), so evacuated or lower density regions stand out as depressions in individual channel maps. The rims of many of these evacuated regions are oval in the low angular resolution ${ }^{13} \mathrm{CO}$ images (see Fig. 4). If molecular gas has been evacuated from a central region, then we would expect that the resulting cavity would appear limb brightened. This appears to be true for a number of cavities evident in the individual channel maps (e.g., C1, C4).

In Figure 4 we have identified and labeled a number of cavities. Their estimated central positions and velocities are listed in Table 1 in order of increasing central velocity. These cavities correspond to depressions in channel maps that we have also identified as depressions in position-velocity diagrams. To list a cavity, we required that they be visible (by eye) as depressions in the intensity seen in both channel maps and position-velocity diagrams; however, we did not require a specific contrast level in the intensity. In some cases the intensity of the edge of the cavity is only $\sim 50 \%$ that of the center in a particular channel, and in other cases the intensity inside the cavity is a few times lower than that of its rim (e.g., C10). We also required that the depression be surrounded on at least three sides by higher intensity emission in a channel map.

In Figure 5 we show position-velocity diagrams extracted from the ${ }^{13} \mathrm{CO}$ velocity cube in narrow horizontal strips. The width of the strip corresponds to 1 pixel in the velocity cube or $25^{\prime \prime}$, which is less than half the beam width. The $x$-axis of these plots is the right ascension (J2000.0), and the $y$-axis the velocity in kilometers per second. Figure 5 shows position-velocity diagrams extracted for a number of different horizontal (eastwest) strips each separated by $2^{\prime}$ in declination. The declination (J2000.0) of each strip is shown in the upper right corner of each subplot. Figure 6 shows a similar set of position-velocity diagrams; however, each plot is extracted along a narrow strip oriented vertically. Consequently, the $x$-axes are declination (J2000.0). The separation in right ascension between strips is $2^{\prime}$. The right
TABLE 1

CAvities Identified IN ${ }^{13} \mathrm{CO}(1-0)$

\begin{tabular}{|c|c|c|c|c|}
\hline Name & $\begin{array}{c}\text { R.A. } \\
\text { (J2000.0) }\end{array}$ & $\begin{array}{c}\text { Decl. } \\
(\mathrm{J} 2000.0)\end{array}$ & $\begin{array}{l}\text { Velocity } \\
\left(\mathrm{km} \mathrm{s}^{-1}\right)\end{array}$ & $\begin{array}{c}\text { Velocity Range } \\
\qquad\left(\mathrm{km} \mathrm{s}^{-1}\right)\end{array}$ \\
\hline $\mathrm{C} 1 \ldots$ & 32914 & +310900 & 5.5 & $5.2-7.0$ \\
\hline $\mathrm{C} 2 \ldots$ & 32829 & +310320 & 6.0 & -6.5 \\
\hline $\mathrm{C} 3 \ldots$ & 32903 & +310550 & 6.1 & $5.5-6.5$ \\
\hline $\mathrm{C} 4 \ldots \ldots \ldots \ldots$ & 32914 & +312300 & 6.3 & -6.8 \\
\hline С5 & 32914 & +311520 & 6.3 & $4.5-7.0$ \\
\hline 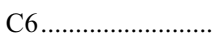 & 32819 & +310150 & 6.8 & $6.5-7.5$ \\
\hline $\mathrm{C} 7 \ldots \ldots \ldots$ & 32929 & +312010 & 6.9 & $6.5-8.0$ \\
\hline C8 & 32832 & +311610 & 7.0 & -7.5 \\
\hline $\mathrm{C} 9 \ldots$ & 32916 & +310600 & 7.3 & $6.5-8.0$ \\
\hline $\mathrm{C} 10 \ldots \ldots$ & 32858 & +311800 & 7.3 & $5.5-10.5$ \\
\hline $\mathrm{C} 11 \ldots \ldots \ldots \ldots$ & 32850 & +310700 & 7.3 & $6.5-7.8$ \\
\hline $\mathrm{C} 12$ & 32851 & +310200 & 7.5 & $6.5-7.8$ \\
\hline $\mathrm{C} 13$ & 32837 & +311130 & 7.6 & $7.0-8.5$ \\
\hline $\mathrm{C} 14 \ldots \ldots \ldots \ldots \ldots \ldots \ldots$ & 32909 & +311200 & 7.7 & $7.0-8.3$ \\
\hline $\mathrm{C} 15 \ldots \ldots \ldots \ldots \ldots \ldots \ldots$ & 32945 & +311210 & 8.0 & $7.0-9.5$ \\
\hline $\mathrm{C} 16 \ldots \ldots \ldots$ & 32925 & +312630 & 8.0 & $7.5-$ \\
\hline $\mathrm{C} 17 \ldots \ldots \ldots \ldots \ldots \ldots \ldots$ & 32910 & +312450 & 8.1 & $7.5-8.5$ \\
\hline $\mathrm{C} 18 \ldots \ldots \ldots \ldots \ldots \ldots \ldots$ & 32922 & +311530 & 8.3 & $7.8-9.0$ \\
\hline $\mathrm{C} 19 \ldots \ldots \ldots \ldots \ldots \ldots \ldots$ & 32951 & +311810 & 9.1 & $8.5-11.0$ \\
\hline $\mathrm{C} 20 \ldots \ldots \ldots \ldots \ldots \ldots$ & 32852 & +311550 & 9.1 & $8.5-$ \\
\hline $\mathrm{C} 21 \ldots \ldots \ldots \ldots \ldots \ldots \ldots$ & 32905 & +311420 & 9.1 & $8.0_{-}$ \\
\hline 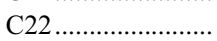 & 32909 & +312410 & 9.3 & $8.5-$ \\
\hline
\end{tabular}

Notes.-Units of right ascension are hours, minutes, and seconds, and units of declination are degrees, arcminutes, and arcseconds. Cavities are labeled in ${ }^{13} \mathrm{CO}(1-0)$ channel maps and position-velocity diagrams and channel maps shown in Figs. 4, 5, and 6. Central cavity right ascension and declination are given with epoch J2000.0. The central velocity is given with respect to the local standard of rest. The rightmost column shows the approximate minimum and maximum velocities of the channels in which the cavity is seen.

ascension (J2000.0) of each strip is shown in the upper right corner of each subplot.

\subsection{Cavity Properties}

The cavities that we have identified in Figures 4, 5, and 6 have typical widths of $2^{\prime}-4^{\prime}$ corresponding to $0.1-0.2 \mathrm{pc}$. The density contrast suggested by the difference between the emission intensity at the center of the cavity and that in the edge-brightened rims is a factor of 2-4. Cavities are evident in channel maps and are also seen as depressions in the position-velocity diagrams. For many cavities, at the cavity center emission can be seen redshifted from the mean central velocity and also blueshifted from this mean. If the cavity is expanding, the redshifted emission we would interpret to be behind the cavity moving away from us and the blueshifted would correspond to material in front of the cavity moving toward us. The width in velocity space of these cavities is approximately $1 \mathrm{~km} \mathrm{~s}^{-1}$ except for extreme cavities such as $\mathrm{C} 10$, which is seen in the channel maps between 6 and $9 \mathrm{~km} \mathrm{~s}^{-1}$. Some cavities are bounded by both redshifted and blueshifted emission (e.g., C11), and others appear to have one open end (e.g., C10).

Many cavities are connected to or associated with other cavities. In other words, they are near in position and velocity to other cavities. For example, C11, C12, and C13 together may form one long tubular region oriented north-south in the cloud. $\mathrm{C} 9$ and $\mathrm{C} 14$ are a smoothly connected triangular region, and $\mathrm{C} 7$ and $\mathrm{C} 4$ could be connected. $\mathrm{C} 15$ and $\mathrm{C} 5$ could be a connected tubular region running southeast. In the southern region of the cloud, the interconnected cavities seem to run north-south, whereas 


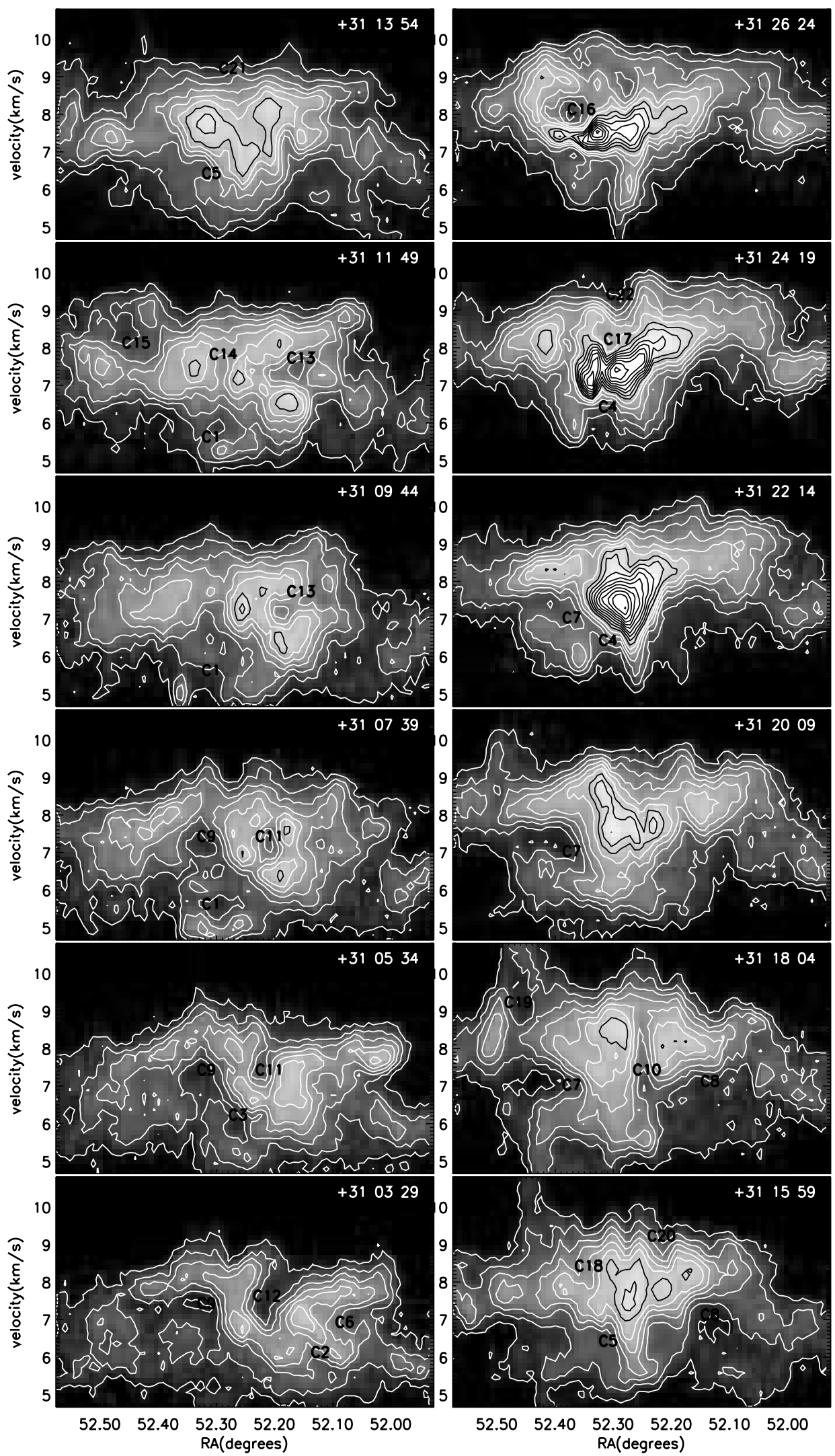

FIG. 5. - Position-velocity maps. Each map is located at a different declination in the ${ }^{13} \mathrm{CO}(1-0)$ velocity cube. This declination is shown in the upper right corner of each panel. Velocity is shown as the vertical axis, whereas the horizontal axes are right ascension (J2000.0). The contour spacing is $0.5 \mathrm{~K}$ with the lowest contour at $0.5 \mathrm{~K}$. Depressions in the intensity identified in both position-velocity diagrams and individual channel maps are labeled here on the channel maps. The properties of these cavities are listed in Table 1. 


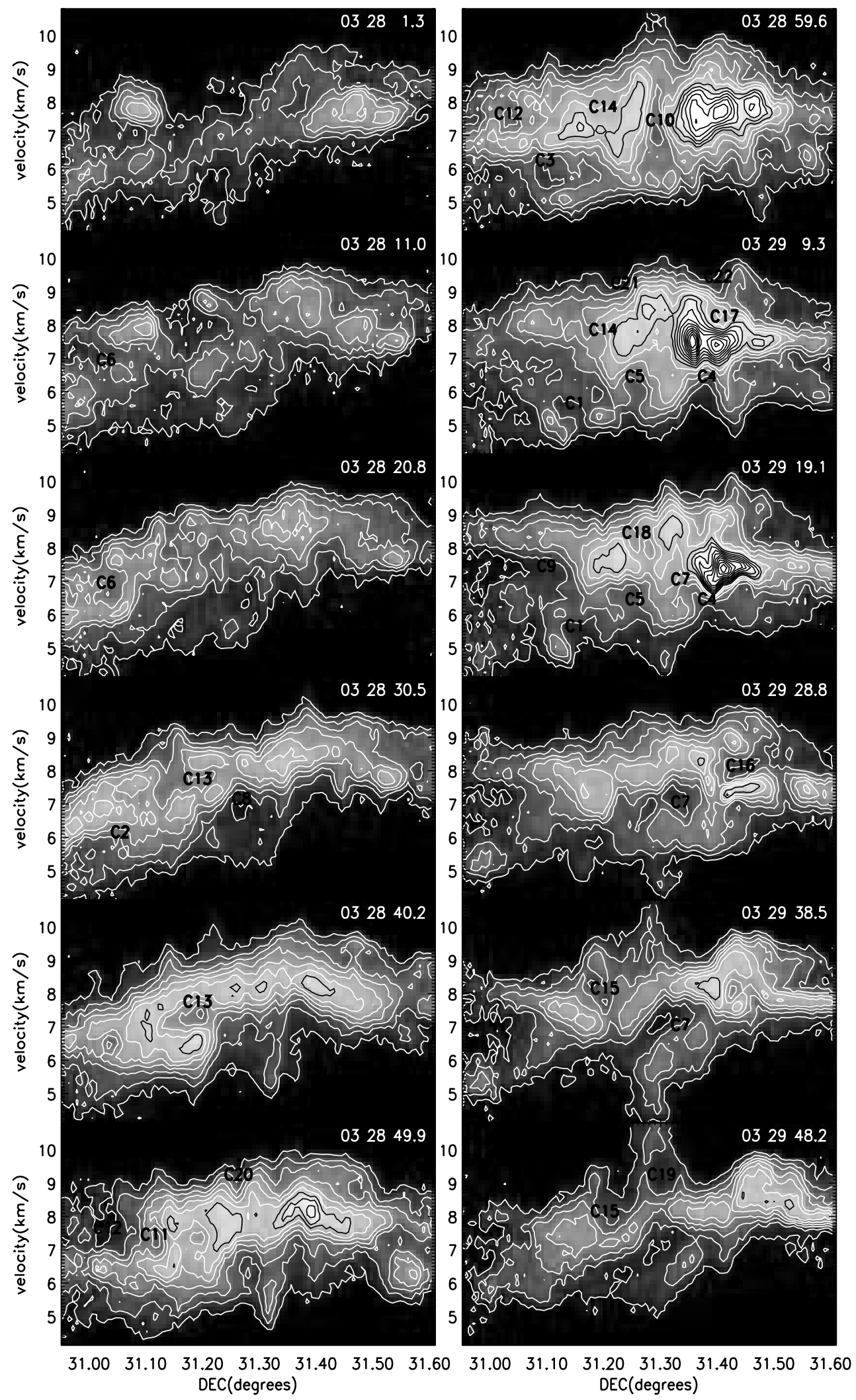

FIG. 6.-Position-velocity maps. Each map is located at a different right ascension in the ${ }^{13} \mathrm{CO}(1-0)$ velocity cube. This right ascension is shown in the upper right corner of each panel. Velocity is shown as the vertical axis, whereas the horizontal axes are declination (J2000.0). The contour spacing is $0.5 \mathrm{~K}$ with the lowest contour at $0.5 \mathrm{~K}$. 
on the eastern side of the cloud, cavities tend to be oriented eastwest. The orientation of the cavities would be consistent with a scenario where the cavities were associated with previous or relic outflows driven from sources near the center of the cloud. The center of the cloud contains two clusters of young stars that are evident in near-infrared images (see Fig. 3). These sources could have driven active outflows a few hundred thousand to about a million years ago (Aspin 2003).

Cavities that have redshifted and blueshifted rims (e.g., C13, $\mathrm{C} 11, \mathrm{C} 9, \mathrm{C} 3, \mathrm{C} 9, \mathrm{C} 8, \mathrm{C} 1, \mathrm{C} 7, \mathrm{C} 16$ ) are most easily interpreted in terms of expansion. The radial expansion rate is slow, $0.5-$ $1 \mathrm{~km} \mathrm{~s}^{-1}$ typically; however, this is high enough that it is above the sound speed for the cold temperatures typical of molecular clouds $\sim 0.3 \mathrm{~km} \mathrm{~s}^{-1}$, although it is similar to the cloud's velocity dispersion. A few of the cavities (e.g., C5, C10, and C19) have larger radial expansion velocities $\left(1.2-2.5 \mathrm{~km} \mathrm{~s}^{-1}\right)$, above the velocity of turbulent motions in the cloud. The redshifted and blueshifted rims would be consistent with a cavity that is slowly expanding in the cloud. Because emission can be brighter in the redshifted or blueshifted rims, compared to nearby material (e.g., C9, C16), the cavities are likely to be expanding rather than boundaries of regions of the cloud that have only been evacuated by outflows.

An expanding spherical shell would appear as an oval symmetrical about the spatial axis in position-velocity diagrams. If the position-velocity diagram is extracted along the major axis of an expanding ovoid shell, the cavity would appear as a skewed oval (one oriented along a diagonal line) in the position-velocity diagram, with one side redshifted and the other side blueshifted (e.g., Welch et al. 2000). If the position-velocity diagram is extracted along a different axis, the cavity would also appear as an oval in the position-velocity diagram but with central position and velocity dependent on the location of the extracted positionvelocity diagram. Since we were searching the entire cloud for cavities, we have extracted in Figures 5 and 6 position-velocity diagrams in north-south and east-west strips in the cloud. If nonspherical (elongated or ovate) cavities are oriented at different positions with respect to the line of sight and the angle that the position-velocity diagrams are extracted, we would expect to see skewed ovals in the position-velocity diagrams, as well as ovals with positions that vary with position in neighboring positionvelocity diagrams (see, e.g., Welch et al. 2000).

In the position-velocity diagrams we see examples of cavities that could be interpreted as expanding nonspherical or ovate shells at different orientations. For example, C10 appears to be oriented nearly along the line of sight. It is a small cavity that spans $3 \mathrm{~km} \mathrm{~s}^{-1}$ and has almost no skew in the position-velocity diagrams. In other words, the cavity central position is not strongly dependent on the velocity channel, and the central velocity does not strongly depend on position on the sky. However, C18 and C19 together may be an example of a cavity that is skewed in the position-velocity diagram, with the western side redshifted and the eastern side blueshifted. The connected cavities (e.g., $\mathrm{C} 11, \mathrm{C} 12, \mathrm{C} 13$ ) could be examples of cavities that change location in both velocity and position in different position-velocity diagrams. It is likely that we are seeing expanding ovoid cavities oriented at different angles with respect to the viewer, as well as those oriented along the line of sight, such as C10. C10 is seen at almost all velocities (except near $5 \mathrm{~km} \mathrm{~s}^{-1}$ ), suggesting that it passes through the entire cloud. Other cavities, such as $\mathrm{C} 12$ and $\mathrm{C} 15$, are seen only at higher or lower velocities, suggesting that they pass through only one side of the cloud.

A few cavities exhibit high-velocity wings (of order $2 \mathrm{~km} \mathrm{~s}^{-1}$ from the mean, which is above the velocity dispersion of the cloud). C10 is extraordinarily broad, extending through a number of channel maps. The cavity has a width of about $3 \mathrm{~km} \mathrm{~s}^{-1}$. C5 could be connected to blueshifted high-velocity gas (at $4 \mathrm{~km} \mathrm{~s}^{-1}$ ); see the panel at R.A. $=3^{\mathrm{h}} 29^{\mathrm{m}} 19^{\mathrm{s}}(\mathrm{J} 2000.0)$ in Figure 6 . The evacuated triangular shape in the position-velocity diagram suggests that a conical-shaped cavity could have been opened. This shape has been seen in previous works. For example, Arce \& Sargent (2004) suggested that IRAS 20582+7724 exhibited a cavity that had been opened by a wide-angle wind. C19 is also a triangular-shaped cavity in the position-velocity diagram (see the panel at decl. $=+31^{\circ} 18^{\prime} 04^{\prime \prime}[\mathrm{J} 2000.0]$ in Fig. 5) and is associated with redshifted gas $2 \mathrm{~km} \mathrm{~s}^{-1}$ above the mean of the cloud. Because they are associated with higher velocity emission on their rims, $\mathrm{C} 10, \mathrm{C} 5$, and $\mathrm{C} 19$ could be examples of younger cavities.

The $\mathrm{C}^{18} \mathrm{O}(1-0)$ velocity cube has lower signal-to-noise ratio than the ${ }^{13} \mathrm{CO}(1-0)$ cube; however, the $\mathrm{C}^{18} \mathrm{O}(1-0)$ line also has lower optical depth. Features detected in ${ }^{13} \mathrm{CO}$ in the outer, more tenuous regions are more difficult to detect in the $\mathrm{C}^{18} \mathrm{O}$ velocity cube. However, cavities identified in the denser regions of the cloud should be visible in both velocity cubes. In Figure 7 we show position-velocity plots of the $\mathrm{C}^{18} \mathrm{O}$ emission extracted in the same regions as those of Figure 5, which displays the ${ }^{13} \mathrm{CO}(1-0)$ emission. From a comparison between Figures 5 and 7 we see that cavities such as C6, C7, C11, C12, C13, C14, and $\mathrm{C} 16$ that have higher intensity rims are seen in both velocity cubes. The cavities that are not detected in the $\mathrm{C}^{18} \mathrm{O}$ velocity cube lie in the more diffuse regions of the cloud. Particularly prominent in the $\mathrm{C}^{18} \mathrm{O}$ position-velocity plot is the cavity $\mathrm{C} 16$. The good correspondence between the morphology seen in these two figures confirms that the cavities identified in ${ }^{13} \mathrm{CO}$ in the denser regions of the cloud are not due to noise in the data and are not artifacts introduced by variations in optical depth.

\subsection{The Relation between Cavities and Infrared Sources}

In this section we compare the location of the cavities seen in individual channel maps to the positions of sources identified from previous surveys. A comparison between the location of cavities and the near-infrared $K_{s}$-band 2MASS images showing $\mathrm{T}$ Tauri stars, young stellar clusters, and background sources is shown in Figure 8. We have plotted our cavity locations along with the positions of sources identified from infrared imaging surveys (Aspin 2003; Lada et al. 1996; Strom et al. 1976), HH objects (Reipurth 1999), and molecular outflows studied by Knee \& Sandell (2000). The positions of the B stars BD +30 547 and BD +30 549 and infrared source SVS 13 are also shown in this figure.

While it was tempting to associate every cavity with a source, no matter how faint, in practice some cavities did not contain sources likely to be responsible for them. For example, cavities on the southern side of the cloud such as $\mathrm{C} 11$ and $\mathrm{C} 12$ have no $\mathrm{HH}$ or 2MASS counterparts. HH objects and embedded YSOs (as seen from the submillimeter observations of Sandell \& Knee 2001) appear to reside within the cloud and are not located in these cavities. The outflows bright in shocked CO and molecular hydrogen and those identified as $\mathrm{HH}$ objects tend to be located along regions of bright ${ }^{13} \mathrm{CO}$ emission rather than located in cavities. For example, IRAS 1 is located at a peak in the ${ }^{13} \mathrm{CO}$ emission in most channel maps rather than a depression in any channel map.

The lack of correspondence between the cavities and molecular outflows, HH objects, and submillimeter and IRAS sources suggests that the cavities are not directly associated with the youngest stellar objects. We now consider the possibility that the cavities are related to older Class II sources that should be visible 

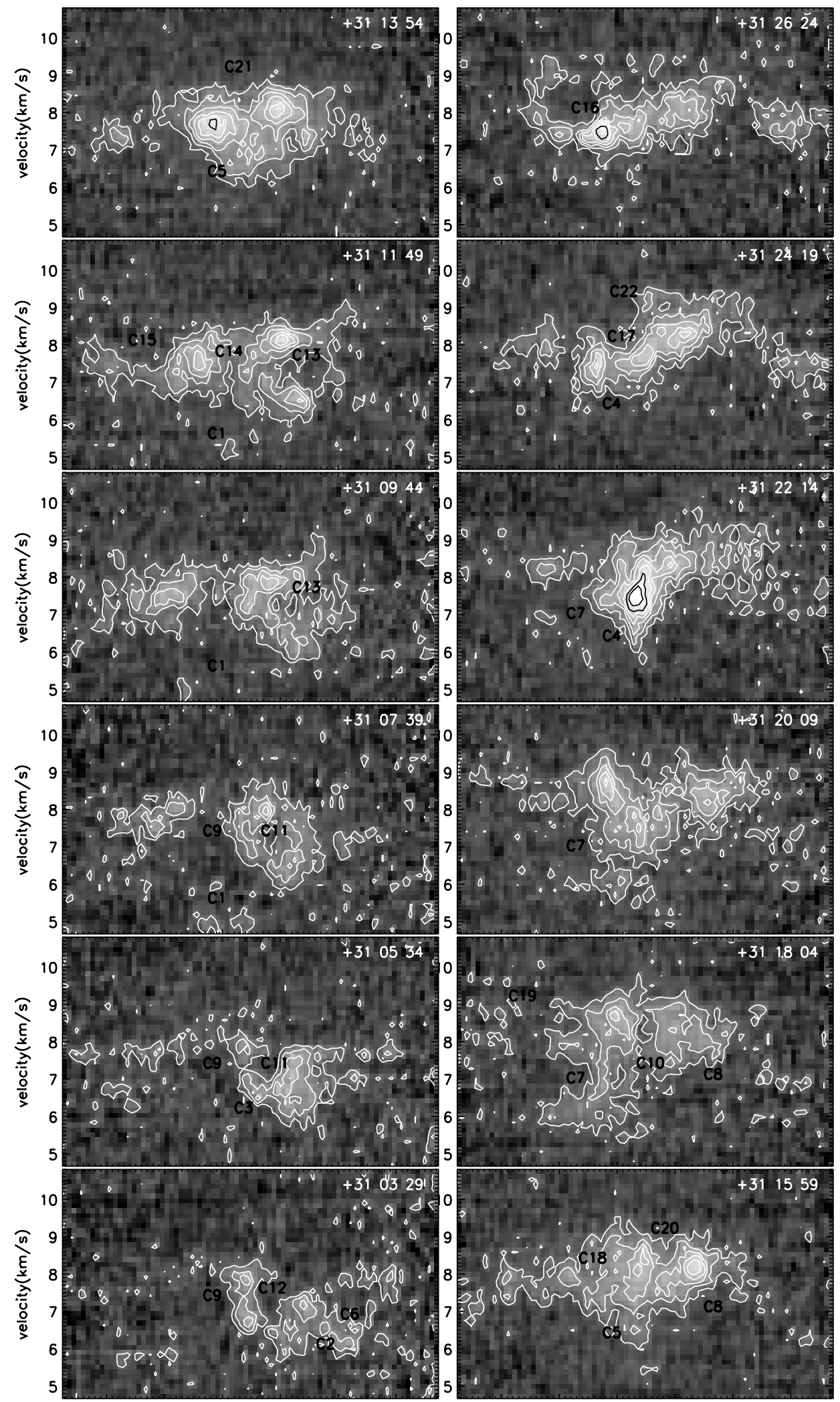

$\begin{array}{ccc}52.50 \quad 52.40 & 52.30 & 52.20 \\ & R A(\text { degrees })\end{array}$

$\begin{array}{llllll}52.50 & 52.40 & 52.30 & 52.20 & 52.10 & 52.00\end{array}$

FIg. 7.-Position-velocity maps from the $\mathrm{C}^{18} \mathrm{O}(1-0)$ velocity cube. Similar to Fig. 5 . The contour spacing is $0.2 \mathrm{~K}$ with the lowest contour at $0.2 \mathrm{~K}$. Cavities in the denser regions of the cloud identified from the ${ }^{13} \mathrm{CO}(1-0)$ velocity cube are also evident in the $\mathrm{C}^{18} \mathrm{O}$ position-velocity maps. 


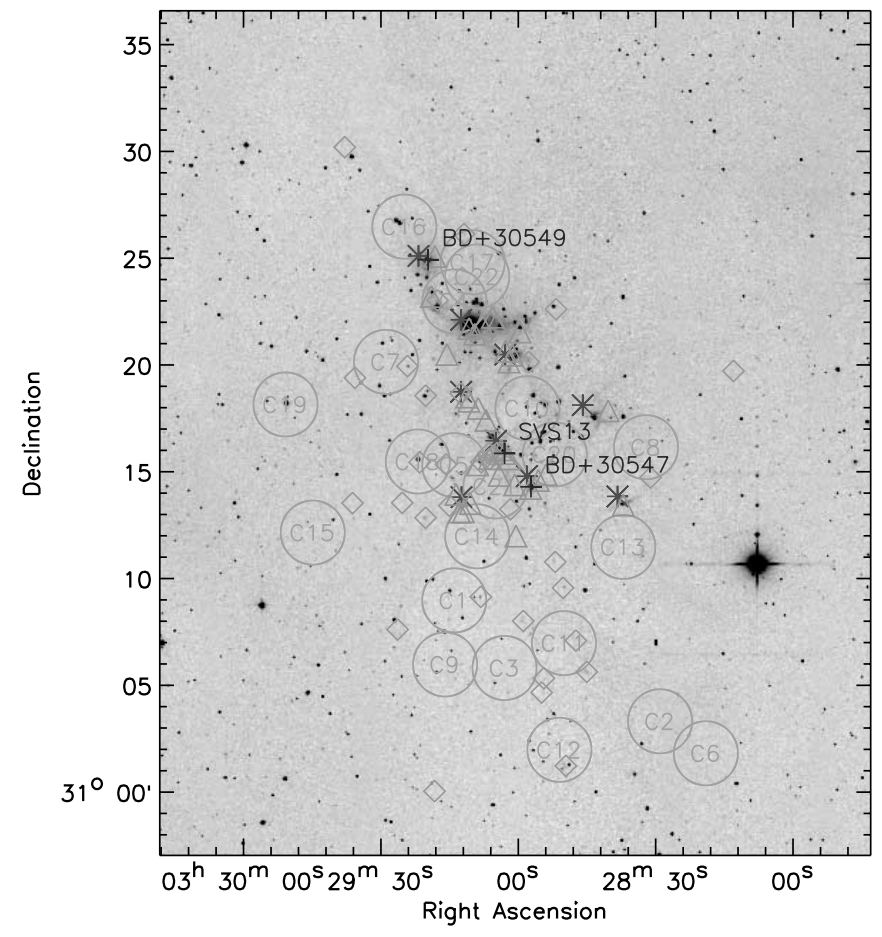

FIG. 8.-Positions of cavities compared to the locations of other sources. IRAS sources from Jennings et al. (1987) are shown by stars. HH objects from Reipurth (1999) are shown by diamonds. Compact submillimeter sources from Knee \& Sandell (2000) are shown by triangles. The cavities are shown by circles of radius 1.5. The gray-scale background is the $K_{\mathrm{s}}$-band 2MASS image and shows T Tauri cluster stars and background stars. The B stars BD +30 547 and BD +30549 and infrared source SVS 13 are shown by crosses. [See the electronic edition of the Journal for a color version of this figure.]

in the 2MASS $K_{s}$-band images. Stars in the stellar clusters evident in the 2MASS images or the infrared imaging surveys of Aspin (2003) and Lada et al. (1996) could be associated with cavities near the center of the cloud. The stars studied by Aspin (2003) and Lada et al. (1996) (identified from near-infrared imaging) have ages of approximately 1 million years old. Because of the large number of the stars seen in $K_{s}$ band on the northern side of the cloud, it would be possible to match each cavity in this region with a pre-main-sequence object. However, the lack of near-infrared objects on the southern and eastern regions of the cloud makes it impossible to associate each cavity with a nearby pre-main-sequence object. While the lack of correspondence between younger sources (embedded with outflows) and cavities implies that the cavities could be relics of previous activity, the lack of correspondence between cavities and older sources (see in the near-infrared images) suggests that cavities could be created distant from the current location of the source originally responsible for driving it.

If the cavities are relics, then the stars that could have caused them may have drifted from their location of birth. T Tauri stars have been estimated to be approximately a million years old (Aspin 2003). A star moving at $1 \mathrm{~km} \mathrm{~s}^{-1}$ (approximately the velocity dispersion of the cloud) would be able to move $0.1 \mathrm{pc}$ in $10^{5} \mathrm{yr}$. This is far enough that it could have moved across a cavity. If the cavities are relics of outflows, their source stars could have moved away from the location where they drove outflows. For example, the embedded Class II source IRAS 16316-1540 lies at the edge of a cavity (associated with RNO 91) that it could have caused (Lee et al. 2002). Cavities could also be relics of long jets (greater than $0.5 \mathrm{pc}$ long) that have punctured tubular holes through the molecular cloud. Thus, cavities could be formed distant from the source originally responsible for driving the jet.

In the previous section we pointed out three cavities (C10, $\mathrm{C} 5$, and $\mathrm{C} 19$ ) that have higher expansion velocities than the others. Since these three cavities are probably younger, it may be easier to find candidate objects responsible for causing them. $\mathrm{C} 5$ and $\mathrm{C} 10$ are located in a region that has been covered by most previous surveys, whereas $\mathrm{C} 19$ is not, making it difficult to search for a candidate object responsible for this cavity. A variety of types of candidate sources are located in the vicinity of C5. C10 contains pre-main-sequence stars but no IRAS source, CO outflow, submillimeter source, or HH object (see Fig. 8). Since C10 is probably pointed toward us, we expect the candidate driving source to be in the vicinity of the cavity. This suggests that $\mathrm{C} 10$ was driven by one of the nearby pre-mainsequence stars and not a younger source with an active outflow.

\subsection{Observational Summary}

Before we discuss explanations and scenarios accounting for the cavities we have identified in NGC 1333 in ${ }^{13} \mathrm{CO}$, we summarize their observational properties:

1. The molecular cloud of NGC 1333 is full of limbbrightened shells and cavities that are evident in both channel maps and position-velocity slices in the ${ }^{13} \mathrm{CO}$ velocity cube. We have identified 22 cavities in an approximately $1 \times 1 \mathrm{pc}$ region on the sky.

2. We can estimate the volume filling factor of these cavities in the cloud. We have 22 cavities with approximate radii of $0.1 \mathrm{pc}$ in a volume approximately $(1 \mathrm{pc})^{3}$. We estimate that approximately $10 \%$ by volume of the molecular cloud is comprised of cavities. Lower rim expansion velocity, lower density contrast, and smaller cavities would be more difficult to detect in the ${ }^{13} \mathrm{CO}$ velocity cube. Since we cannot have identified every cavity in this cloud, we can regard the estimated filling factor as a lower limit. We conclude that cavities permeate the molecular cloud.

3. Limb brightening is seen in both position space (making shells) and velocity space, suggesting that cavities can be expanding.

4. Cavities have expansion velocity widths of $\sim 1 \mathrm{~km} \mathrm{~s}^{-1}$ and spatial widths of $0.1-0.2 \mathrm{pc}$. The intensity contrast between that at the rim and that in the center is not high, $\sim 2-4$, as estimated from a comparison between the emission intensity in the cavity center at the cavity's central velocity and the emission temperature at its edge. If the edges of the cavities are turbulent, then the edge would cause emission at a range of velocities. Consequently, the true density contrast between the density at the rim and that inside the cavity could be larger than our measured factor.

5. Cavities appear to be nonspherical. They could be ovate or cylindrical. Examples of elongated cavities at different orientations are seen in the velocity cube.

6 . We see three cavities with higher velocity ${ }^{13} \mathrm{CO}$ emission (above $2 \mathrm{~km} \mathrm{~s}^{-1}$ from the cloud mean), suggesting that some cavities could be younger than others. There could be an evolutionary sequence.

7. Some cavities appear to be connected to others. Tubular cavities are oriented north-south on the southern part of the cloud and east-west on the eastern side. This suggests that these cavities could have been driven by previously active outflows located near the center of the cloud where there are million year old star clusters. 
8. Cavities are not directly or obviously associated with outflows previously identified as $\mathrm{HH}$ objects or seen in shocked molecular hydrogen and CO in Spitzer $4.5 \mu \mathrm{m}$ images (A. Porras 2004, private communication). Instead, these YSOs seem to be associated with dense molecular regions or filaments that are emitting (rather than deficient) in ${ }^{13} \mathrm{CO}$.

Cavity expansion velocities $\left(\sim 1 \mathrm{~km} \mathrm{~s}^{-1}\right)$ are observed to be above the sound speed of the cloud, although they are similar to the size of the cloud's velocity dispersion. For gas traveling at the sound speed of a fraction of a kilometer per second typical of a cold molecular cloud, it would take a few times $10^{5} \mathrm{yr}$ for a $0.2 \mathrm{pc}$ diameter cavity to fill in if the cavity were not expanding and assuming that currently there is no momentum source inside the cavity adding to the expansion. Turbulent motions in the cloud could lower this timescale by a factor of a few. However, it might take an additional few times $10^{5} \mathrm{yr}$ longer to fill in the cavity taking into account the need to overcome the momentum in the walls implied from the estimated expansion velocities. Based on the age of the cluster, estimated from the age of the oldest cluster members or a few million years, the cavity lifetime is short with respect to the age of the entire system. This suggests that the cavities have formed fairly recently, even though they do not seem to be directly associated with existing outflows.

Our failure to match cavities with existing outflows suggests that the cavities identified here are primarily relics and associated with past outflow activity, as previously suggested by Bally et al. (1999). Whereas existing outflows do not seem to be strongly perturbing the bulk of the cloud, the cavities we identify here permeate the cloud. It is possible that we failed to match individual outflows to the cavities identified here in part because of the low angular resolution of the ${ }^{13} \mathrm{CO}$ velocity cube in comparison to other studies (e.g., Arce \& Sargent 2004). The ${ }^{13} \mathrm{CO}$ velocity cube used here is only sensitive to cavities larger than $\sim 0.05 \mathrm{pc}$ since the spatial resolution of these data is $47^{\prime \prime}$ (Ridge et al. 2003), corresponding to $\sim 0.05 \mathrm{pc}$ at the distance of NGC 1333. We expect that cavities with diameter smaller than $0.1 \mathrm{pc}$ would not only be harder to detect in the velocity cube studied here but might also be shorter lived, making them rarer. In short, smaller cavities could also be associated with molecular outflows and $\mathrm{HH}$ objects, but we may not be able to detect them in the ${ }^{13} \mathrm{CO}$ cube studied here if they are too small.

It is possible that the cavities we have identified here are a result of random turbulence that is excited by energy sources external to the cluster. For example, simulations of supersonic compressible MHD turbulence in a sheering disk can show depressions in synthetic channel maps (see, e.g., Fig. $2 b$ of Pichardo et al. 2000 ) that mimic the signature of an expanding cavity. Differentiating between organized structures such as shells, cavities, and filaments and those caused by random turbulent motions is a pervasive problem in the study of the interstellar medium (ISM). For example, Elmegreen \& Scalo (2004) at the end of their recent review of interstellar turbulence ask, "Why is the power spectrum of density structure a power law when direct observation shows the ISM to be a collection of shells, bubbles, comets, spiral wave shocks and other pressurized structures spanning a wide range of scales?"

To differentiate between structures generated from sources within the cloud and those arising from turbulent motions (with energy source assumed to be external to the cloud), we can attempt to search for differences between the structures seen in the velocity cube of NGC 1333 and those exhibited by simulations of turbulent media. We qualitatively compare Figure 4 to the synthetic channel maps shown in Figure $2 b$ of Pichardo et al. (2000). Depressions in the synthetic channel maps tend to have redshifted or blueshifted rims, but not both (as do C11, $\mathrm{C} 13$, and $\mathrm{C} 16$ ). Depressions in the gas density that span a range of velocities larger than the velocity dispersion of the cloud (such as C5, C10, and C19) appear to be absent in the simulation. This comparison suggests that conical-shaped evacuated regions might be more easily confused with structures created by turbulence; however, ovate or spherical or higher expansion velocity cavities are likely to have been driven by internal energy sources. This comparison suggests that some (but not necessarily all) of our candidate cavities have been driven by energy sources internal to the cloud. But if high-velocity cavities are likely to have been driven by internal sources, then we infer that there should also be lower velocity structures that correspond to older or weaker expanding cavities. Likewise, if cavities with both redshifted and blueshifted rims are likely to have been driven by internal sources, then we infer that there should also be cavities located at the edge of the cloud that have prominent rims only on one side. The simulation of Pichardo et al. (2000) illustrates general properties of supersonic compressible MHD turbulence driven by energy at large scales; however, the scale of the simulation $(500 \mathrm{pc})$ is larger than that of our cloud (1 pc) and so the simulation is not an ideal physical analogy to NGC 1333. It may in future be possible to develop better tools to differentiate between the scenarios for producing structures observed in molecular clouds.

\section{ENERGETICS OF CAVITIES}

To investigate the energetics of driving cavities, we consider wind-blown bubble models (see, e.g., the review by Frank 1999). When the bubble is fully radiative, we can consider the momentum-conserving case (e.g., Koo \& McKee 1992a, $1992 b$ ). We assume that the outflow responsible for the cavity has ceased, consistent with the interpretation of these cavities as relics. Therefore, we wish to relate the size and expansion velocity of the cavity to the total momentum imparted to the ambient cloud from the outflow. By dimensional analysis in the spherical case

$$
\frac{P_{w}}{\rho} \sim R^{4} t^{-1}
$$

where $P_{w}$ is the total momentum output during the lifetime of the outflow; $P_{w}=\dot{M}_{w} V_{w} \tau_{w}$, where $\dot{M}_{w}$ is the mass flux in the wind at a mean velocity $V_{w}$, during a lifetime $\tau_{w}$. Here $R$ is the radius of the cavity at time $t$ and $\rho$ is the cloud density. This equation is similar to the Sedov solution for an explosion and is appropriate if the hydrodynamics can be described by scale-free functions. More detailed one-dimensional spherical models (e.g., Koo \& McKee 1992a, 1992b) support the type of order-of-magnitude scale-free estimate illustrated here. In more convenient units we find

$$
P_{w} \sim 0.2 M_{\odot} \mathrm{km} \mathrm{s}^{-1}\left[\frac{n\left(\mathrm{H}_{2}\right)}{10^{4} \mathrm{~cm}^{-3}}\right]\left(\frac{R}{0.1 \mathrm{pc}}\right)^{4}\left(\frac{t}{2 \times 10^{5} \mathrm{yr}}\right)^{-1},
$$

where we have adopted a number density of molecular hydrogen $n\left(\mathrm{H}_{2}\right) \sim 10^{4} \mathrm{~cm}^{-3}$ typical of the NGC 1333 cloud. Note that this estimate is very sensitive to the radius of the cavity. If the cavity is one-half the size, then the momentum requirements drop by more than an order of magnitude. One can also consider 
the role of the cavity age in shaping the cloud. If we assume that all cavities are produced by outflows of identical momentum outputs, then cavities that differed by a factor of 2 in size would differ by a factor of 16 in age.

For equation (1) we have assumed a spherical model. We can consider a cylindrical cavity with the scaling

$$
\frac{P_{w, \perp}}{\rho l}=R^{3} t^{-1},
$$

where $P_{w, \perp}$ is the total momentum exerted in the direction perpendicular to a jet along the jet axis a length $l$, and $P_{w, \perp} / l$ is the total momentum exerted perpendicular to the jet axis per unit length. Here $l$ refers to the length of the cylinder, which could be half the width of the cloud if the driving source is located at the center of the cloud and the jet has drilled right through the cloud. Using the above scaling relation, we would estimate

$$
\begin{aligned}
P_{w, \perp} \sim & 0.8 M_{\odot} \mathrm{km} \mathrm{s}^{-1}\left(\frac{n}{10^{4} \mathrm{~cm}^{-3}}\right) \\
& \times\left(\frac{R}{0.1 \mathrm{pc}}\right)^{3}\left(\frac{l}{0.4 \mathrm{pc}}\right)\left(\frac{t}{2 \times 10^{5} \mathrm{yr}}\right)^{-1},
\end{aligned}
$$

where we have used parameters similar to those in equation (2).

Both of the above scaling relations imply that the cavity expands at a velocity $v_{\exp }=d R / d t \sim R / t$. Different scaling factors $\left(\frac{1}{3}\right.$ or $\left.\frac{1}{4}\right)$ would be inserted in front of this relation, depending on the power of $R$ in equations (1) and (3). If the cavities in NGC 1333 's molecular cloud are $\sim 2 \times 10^{5}$ years old, we would then predict an expansion velocity close to but somewhat smaller than that observed. Consequently, we adopt $t \sim 2 \times 10^{5} \mathrm{yr}$ since the outflow ceased for the age of the $0.1 \mathrm{pc}$ sized cavities in NGC 1333.

We expect that the ratio of momentum exerted perpendicular to the jet axis to that exerted along the jet axis would depend on a number of quantities. For a collimated jet with a constant thrust, we expect that this ratio would primarily depend on the Mach number of the jet. However, this ratio could also depend on the ratio of momentum originating from a disk wind compared to that originating from a collimated jet. YSOs probably generate outflows that decay with time. Consequently, the bow shock would slow down as a function of time. The width of the cavity compared to its length could increase with time. The cavity's shape, expansion rate at its edges, and density contrast (inside compared to outside) should be sensitive to the history of the outflow responsible for driving it.

A wind-blown cavity model for these cavities can be used to predict observable cavity properties. Using equation (2), we can substitute for the expansion velocity $v_{\exp } \sim R / t$, finding

$$
P_{w} \sim \rho R^{3} v_{\exp },
$$

where we have placed the observables on the right-hand side and left the total momentum required on the left-hand side. The observables are the density $\rho$, the cavity size $R$, and the expansion velocity $v_{\text {exp }}$. Using equation (4) and making the same substitution, we find

$$
P_{w, \perp} \sim \rho l R^{2} v_{\exp }
$$

From these relations we see that a cavity's environment could affect its dynamic properties. A similar sized cavity in a cloud of higher density should be older than a similar size one in a lower density region and so should have a lower expansion velocity. Because it is easier to detect large fast cavities in a velocity cube, we suspect that cavities will be easiest to identify at the edges of clouds. At cloud edges, the lower densities will allow the cavities to grow to a large size while maintaining an expansion velocity below the channel spacing or that of turbulence in the cloud. This does appear to be the case in NGC 1333; see Figure 4, where it is particularly easy to identify large cavities near the cloud edge (e.g., C19).

We also expect scaling relations between cavity observables. Older cavities should be larger and have lower expansion velocities and lower density contrasts. We hope that future studies that aim to match wind-blown bubble models to young cavities (with the highest velocities) can better constrain the total momentum requirements, as well as the nature of the momentum injection, e.g., wide-angle wind or jet, and momentum injection as a function of time (A. Cunningham \& A. Frank 2005, in preparation; S. Thorndike et al. 2005, in preparation).

\subsection{Comparison of Momentum Required to Open Cavities to That in Observed Outflows}

We compare the momentum required to open the cavity (estimated from eq. [2]) to that estimated from young stellar outflows. YSOs (Class 0 and 1 sources) have outflows lasting $\sim 10^{5} \mathrm{yr}$. Momentum fluxes, estimated from observations of entrained material, range from 0.1 to $1000 M_{\odot} \mathrm{km} \mathrm{s}^{-1}$ for low- and highmass objects, respectively (Richer et al. 2000; Fukui et al. 1993). In NGC 1333 itself, Knee \& Sandell (2000) measured a total momentum, due to about six mostly Class 0 sources, of $\sim 10 M_{\odot} \mathrm{km} \mathrm{s}^{-1}$ in swept-up high-velocity gas (here high velocity means greater than a few kilometers per second from the mean). The momentum imparted by the individual outflows ranged from 0.2 to $2 M_{\odot} \mathrm{km} \mathrm{s}^{-1}$. We find that these total momentum values are similar to those estimated from equations (2) and (4) using our scaling relations. Knee \& Sandell (2000) measured energies of $\sim 10^{43}-10^{44}$ ergs in each outflow, which had typical velocities of $10 \mathrm{~km} \mathrm{~s}^{-1}$ based on redshifted and blueshifted ${ }^{12} \mathrm{CO}$ emission.

The momentum traced in the ${ }^{12} \mathrm{CO}$ by Knee \& Sandell (2000) corresponds to sources that are younger than the estimated ages of the cavities. As the YSOs age, we expect that more momentum will be put into the molecular cloud. The rough correspondence between the momentum required to open the cavity and that observed in entrained higher velocity ${ }^{12} \mathrm{CO}$ suggests that a substantial fraction of the momentum from outflows observed in ${ }^{12} \mathrm{CO}$ is required to form the cavities we have identified in the ${ }^{13} \mathrm{CO}$ velocity cube. It may be that only a fraction of the momentum from outflows is actually ejected from the cloud. Thus, we conclude that a degree of momentum coupling exists between the outflows and the cloud. The difference in number of outflows identified in ${ }^{12} \mathrm{CO}$ (handful) and the number of cavities we have identified here (22) implies that cavities can be detected over a timescale that is a few times longer than that for outflows in ${ }^{12} \mathrm{CO}$.

It is likely that there is only a moderately short window in time when a cavity would be identified in the ${ }^{13} \mathrm{CO}$ channel maps. Cavities with radii smaller than $0.05 \mathrm{pc}$ would be difficult to detect with the $47^{\prime \prime}$ beam of the ${ }^{13} \mathrm{CO}$ data. Larger and older cavities would be more slowly expanding and would have lower density contrasts. This would make them more difficult to identify in the channel maps. Based on equation (5), we estimate that a cavity 4 times larger could be moving only half as fast. Thus, we expect that a cavity with a $0.4 \mathrm{pc}$ diameter would have a shell velocity of only $0.2 \mathrm{~km} \mathrm{~s}^{-1}$, at which time it would become 
confused with turbulence in the cloud and thus difficult to identify in an individual channel map. (Note that the channel maps here have a comparable velocity resolution of $0.133 \mathrm{~km} \mathrm{~s}^{-1}$.) Above we have estimated an age of $\sim 2 \times 10^{5}$ yr for a $0.1 \mathrm{pc}$ radius cavity. Equation (6) implies that this cavity would have about one-fourth the expansion velocity when it is twice as big, at which time it would be about 8 times older (using eq. [4]). From this we estimate that an expanding cavity would be detectable in the channel maps during a timescale of about a million years. This estimated timescale compared to that for outflows (a hundred thousand years) could account for the difference in number of outflows identified in ${ }^{12} \mathrm{CO}$ and the number of cavities we have identified in ${ }^{13} \mathrm{CO}$.

\subsection{Excitation of Turbulence}

Previous studies have suggested that outflows from YSOs are sufficiently energetic or contain sufficient momentum that they could account for a significant fraction of turbulence in molecular clouds. Here we have introduced an intermediate stage, the formation of a cavity that is caused by an extinct or previously active outflow. In this subsection we compare the total momentum required to account for the observed cavities to that present in the turbulent cloud.

The momentum and energy injection rate from outflows in NGC 1333 has been estimated to be $10^{-4} M_{\odot} \mathrm{km} \mathrm{s}^{-1} \mathrm{yr}^{-1}$ and $0.1 L_{\odot}$ from the outflows seen in ${ }^{12} \mathrm{CO}$ (Knee \& Sandell 2000). In the previous sections we found that the momentum required to form the observed cavities is $\sim 1 M_{\odot} \mathrm{km} \mathrm{s}^{-1}$, similar to that of a typical outflow seen in ${ }^{12} \mathrm{CO}$ by Knee $\&$ Sandell (2000). There are a few times as many cavities $(22)$ as outflows $(\sim 6)$ seen in ${ }^{12} \mathrm{CO}$. The number of observed cavities is consistent with the longer lifetime we have estimated for these cavities or a few hundred thousand years. Consequently, the total momentum input into the cloud via the expansion of cavities is similar to that estimated from the observed outflows in ${ }^{12} \mathrm{CO}$ or $\sim 10^{-4} M_{\odot} \mathrm{km}$ $\mathrm{s}^{-1} \mathrm{yr}^{-1}$. The total energy flux consistent with the cavities is also similar to that estimated by Knee \& Sandell (2000) or $\sim 0.1 L_{\odot}=4 \times 10^{32}$ ergs.

We note that while we can detect cavities everywhere in the cloud, the opacity of the ${ }^{12} \mathrm{CO}$ line makes it difficult for lower velocity or weak outflows to be detected. For example, some $\mathrm{HH}$ objects are not seen with high-velocity ${ }^{12} \mathrm{CO}$ counterparts. Also the area of the sky covered by Knee \& Sandell (2000) is smaller than that of our ${ }^{13} \mathrm{CO}$ velocity cube and did not include sources such as IRAS 1 because of this. It is possible that the total momentum budget from outflows is somewhat higher than that estimated by Knee \& Sandell (2000).

We now compare the energy flux from outflows into the cloud to that being dissipated in the cloud. Since turbulent energy decays in roughly a crossing time (Elmegreen \& Scalo 2004 and references therein), the energy dissipated by a turbulent medium can be estimated as

$$
L_{\text {turb }} \sim \frac{M_{\text {cloud }} v_{\text {turb }}^{3}}{l_{\text {eddy }}},
$$

where $l_{\text {eddy }}$ is the size of the largest turbulent eddies. Inserting $M_{\text {cloud }}=520 M_{\odot}$ for the mass of the cloud (using that measured by Ridge et al. [2003] but correcting for distance), $v_{\text {turb }} \sim 1 \mathrm{~km} \mathrm{~s}^{-1}$, and $l_{\text {eddy }} \sim 0.3 \mathrm{pc}$, we estimate a total energy dissipation rate of $L_{\text {turb }} \sim 10^{33} \mathrm{ergs} \mathrm{s}^{-1}$. We insert $l_{\text {eddy }} \sim 0.3$ pc corresponding to the approximate size of the filaments on the cloud's southern side. The estimated energy dissipation rate is close to that available from the outflows seen in ${ }^{12} \mathrm{CO}$ and that inferred from the cavities. This confirms previous studies that have found that outflows could be responsible for driving a significant fraction of molecular cloud turbulence (Bally et al. 1996a; Reipurth \& Bally 2001; Knee \& Sandell 2000; Warin et al. 1996). However, here we have provided an additional intermediate step between the driving of the outflow and the excitation of the turbulence, the slow expansion of multiple cavities that have been opened by now extinct YSO outflows.

There is an additional test we can carry out. Previously we estimated the volume filling factor of the cavities to be $\sim 10 \%$. We also have predicted how the cavities evolve in time. We ask, what would the cavity expansion velocity be when it reaches a size large enough to overlap a nearby cavity? When do random turbulent motions in the cloud begin to destroy the cavities? It may not be a coincidence that the cavity expansion velocities $\sim 1 \mathrm{~km} \mathrm{~s}^{-1}$ are similar to the rms velocity dispersion of the cloud. From equation (5) we find that for a given momentum impulse and ambient density, the expansion velocity is only weakly dependent on the cavity size, $v_{\exp } \propto R^{1 / 3}$. Since the cavity expansion velocity is only weakly dependent on the cavity size, the expansion velocity would be similar for larger cavities. The cavities, when they become overlapping, would have expansion velocities near $1 \mathrm{~km} \mathrm{~s}^{-1}$, which is approximately the same as the cloud rms velocity or velocity dispersion. Since this velocity is similar to the random component of the cloud, the cavities will be destroyed by the turbulence of the cloud, which they in fact are contributing to. The high volume filling factor of these cavities and similar expansion velocity to the rms velocity dispersion of the cloud also suggest that they are a significant contributor to the cloud turbulence.

\section{SUMMARY AND DISCUSSION}

In this paper we began by studying the structure of the integrated properties of the ${ }^{13} \mathrm{CO}$ velocity cube in comparison with the distribution of YSOs seen at near-infrared wavelengths. The velocity dispersion of the molecular cloud is between 1 and $1.6 \mathrm{~km} \mathrm{~s}^{-1} \mathrm{rms}$, varies little across the cloud, and does not seem to be closely related to the distribution of YSOs. The mean velocity does show structure, with the southern half of the cloud at a mean velocity that is about $0.3 \mathrm{~km} \mathrm{~s}^{-1}$ below that of the northern half. Little evidence for high-velocity gas is seen in the ${ }^{13} \mathrm{CO}$ velocity cube near the large concentration of YSOs or outflows identified from other studies.

More careful inspection of the ${ }^{13} \mathrm{CO}$ velocity cube reveals what appear to be numerous cavities and shells. These depressions are likely to be real because they are seen in multiple channels, are present in both channel maps and position-velocity diagrams, and some are also seen in the lower signal-to-noise ratio $\mathrm{C}^{18} \mathrm{O}$ velocity cube. Rims are limb brightened in individual channel maps but also tend to contain brighter gas at higher and lower velocities than the mean. The features in the positionvelocity diagrams suggest that the cavities may not be spherical. They could be expanding ovate or cylindrical structures. Examples of cavities likely to be at different orientations are seen. Typical cavity sizes are $0.1-0.2 \mathrm{pc}$ in diameter and have velocity widths $1-3 \mathrm{~km} \mathrm{~s}^{-1}$. From the channel maps we estimate that the density contrast between gas outside and inside the cavity is low, only a factor of 2-4. However, the true density contrast could be higher if the edges of the cavities are turbulent and so contain gas at a range of velocities. We have identified 22 cavities in a $1 \times 1 \mathrm{pc}$ region on the sky. We estimate their volume filling factor to be $\sim 10 \%$. They permeate the molecular cloud. 
We find three cavities with higher velocity ${ }^{13} \mathrm{CO}$ emission (above $2 \mathrm{~km} \mathrm{~s}^{-1}$ from the cloud mean), suggesting that some cavities could be younger than others. Some cavities appear to be connected to others. Tubular cavities are oriented north-south on the southern part of the cloud and east-west on the eastern side. These cavities could have been driven by previously active outflows located near the center of the cloud where there are million year old star clusters.

We have had difficulty matching cavities with YSOs that could have been responsible for opening the cavity. Cavities are not seen near $\mathrm{HH}$ objects or objects identified as outflows from previous surveys. Neither do the cavities appear to be clearly associated with million year old pre-main-sequence objects that would be present in the 2MASS $K_{s}$-band images. We estimate that the timescale for a cavity to fill in at the sound speed or at the random turbulent cloud velocity would not be long, less than a million years, thus the cavities must have been produced within the past million years. If cavities are relics associated with previous outflow activity, then they could be located distant from their driving source. Also their source stars could have drifted from their original location. A star moving $1 \mathrm{~km} \mathrm{~s}^{-1}$ drifts out of a $0.1 \mathrm{pc}$ sized cavity associated with its formation in only $10^{5} \mathrm{yr}$.

From scaling laws, we find that expanding bubbles driven by a jet impulse can match the rough physical properties of the cavities, i.e., their expansion velocities and sizes. The cavities are most likely relics associated with previous outflow activity, as previously suggested by Bally et al. (1999) in the case of the Circinus cloud. We use the dimensional scaling relations to estimate the age and jet/wind impulse total momentum required to make a cavity. This total momentum is about $1 M_{\odot} \mathrm{km} \mathrm{s}^{-1}$, and the estimated cavity age is a few hundred thousand years. The age estimate is approximately consistent with the number of cavities, $\sim 20$, and the number of active outflows in the cloud seen at higher velocities in ${ }^{12} \mathrm{CO}$.

From a comparison between the momentum flux estimated from active outflows measured from higher velocity (greater than a few kilometers per second from the mean) ${ }^{12} \mathrm{CO}$ by Knee \& Sandell (2000) and the requirements to open the cavities, we estimate that most of momentum from outflows is fed back into the cloud. This total mechanical energy is a significant fraction of that required to power the cloud turbulence. However, here we have provided an additional intermediate step between the driving of the outflow and the excitation of the turbulence: the slow expansion of multiple cavities that have been opened by now extinct YSO outflows. The similarity between the cavity expansion velocity widths and the cloud velocity dispersion also suggests that the cavities could form an intermediate step between the outflows and the excitation of the cloud turbulence.

Our estimates of the momentum requirements of these cavities are based on rough order-of-magnitude scaling arguments. Our preliminary simulations have been restricted to jet impulses, rather than jets followed by the slow opening of a cavity. Better models of a longer interaction between jets and winds from YSOs with the cloud may allow better constraints on the properties of the cavities and on how they impact the evolution of the molecular cloud. Future studies can explore in more detail the types of wind-blown models that could account for these cavities and, by fitting observations (channel maps) of individual cavities, better constrain the momentum requirements, type of injection, and outflow evolution (S. Thorndike et al. 2005, in preparation; A. Cunningham \& A. Frank 2005, in preparation).

We thank John Bally, Mike Jura, Tom Meageath, and Alicia Porras for helpful discussions and correspondence. We thank the Spitzer IRAC GTO team for showing us their Spitzer IRAC images in advance of publication. We thank Eve Ostriker and Alyssa Goodman for helpful discussions on turbulence. We acknowledge the hospitality of the Aspen Center for Physics during 2004 July.

Support for this work was in part provided by National Science Foundation grants AST 97-02484, AST 00-98442, AST 0406799, and AST 04-06823, the National Aeronautics and Space Administration under grant NNG04GM12G issued through the Origins of Solar Systems Program, and grant NAG5-8428. Support was also provided by DOE grant DE-FG02-00ER54600, the Laboratory for Laser Energetics, and by the National Science Foundation to the Kavli Institute for Theoretical Physics under grant PHY99-07949.
Arce, H. G., \& Goodman, A. A. 2002a, ApJ, 575, 911 2002b, ApJ, 575, 928

Arce, H. G., \& Sargent, A. I. 2004, ApJ, 612, 342

Aspin, C. 2003, AJ, 125, 1480

Aspin, C., Sandell, G., \& Russell, A. P. G. 1994, A\&AS, 106, 165

Bally, J., Devine, D., \& Alten, V. 1996a, ApJ, 473, 921

Bally, J., Devine, D., \& Reipurth, B. 1996b, ApJ, 473, L49

Bally, J., Reipurth, B., Lada, C. J., \& Billawala, Y. 1999, AJ, 117, 410

Bence, S. J., Richer, J. S., \& Padman, R. 1996, MNRAS, 279, 866

Bergin, E. A., Alves, J., Huard, T., \& Lada, C. J. 2002, ApJ, 570, L101

Cernicharo, J., \& Reipurth, B. 1996, ApJ, 460, L57

Cernis, K. 1990, Ap\&SS, 166, 315

Chernin, L., Masson, C., Gouveia dal Pino, E. M., \& Benz, W. 1994, ApJ, 426, 204

Chernin, L. M., \& Masson, C. R. 1995, ApJ, 455, 182

Delamarter, G., Frank, A., \& Hartmann, L. 2000, ApJ, 530, 923

de Zeeuw, P. T., Hoogerwerf, R., de Bruijne, J. H. J., Brown, A. G. A., \& Blaauw, A. 1999, AJ, 117, 354

Elmegreen, B. G., \& Scalo, J. 2004, ARA\&A, 42, 211

Frank, A. 1999, NewA Rev., 43, 31

Fukui, Y., Iwata, T., Mizuno, A., Bally, J., \& Lane, A. P. 1993, in Protostars and Planets III, ed. E. H. Levy \& J. I. Lunine (Tucson: Univ. Arizona Press), 603

Gardiner, T. A., Frank, A., \& Hartmann, L. 2003, ApJ, 582, 269

\section{REFERENCES}

Jennings, R. E., Cameron, D. H. M., Cudlip, W., \& Hirst, C. J. 1987, MNRAS, 226,461

Knee, L. B. G., \& Sandell, G. 2000, A\&A, 361, 671

Koo, B.-C., \& McKee, C. F. 1992a, ApJ, 388, 93 1992b, ApJ, 388, 103

Lada, C. J., Alves, J., \& Lada, E. A. 1996, AJ, 111, 1964

Lee, C.-F., Mundy, L. G., Stone, J. M., \& Ostriker, E. C. 2002, ApJ, 576, 294

Lefloch, B., Castets, A., Cernicharo, J., Langer, W. D., \& Zylka, R. 1998, A\&A, 334, 269

Myers, P. C., \& Fuller, G. A. 1992, ApJ, 396, 631

Noriega-Crespo, A., et al. 2004, ApJS, 154, 352

Pichardo, B., Vazquez-Semadeni, E., Gazol, A., Passot, T., \& BallesterosParedes, J. 2000, ApJ, 532, 353

Raga, A., \& Cabrit, S. 1993, A\&A, 278, 267

Raga, A. C., Noriega-Crespo, A., Gonzalez, R., \& Velazquez, P. F. 2004, ApJS, 154,346

Reipurth, B. 1999, A General Catalog of Herbig-Haro Objects, 2nd ed., http:// casa.colorado.edu/hhcat

Reipurth, B., \& Bally, J. 2001, ARA\&A, 39, 403

Reipurth, B., Devine, D., \& Bally, J. 1998, AJ, 116, 1396

Richer, J., Shepherd, D., Cabrit, S., Bachiller, R., \& Churchwell, E. 2000, in Protostars and Planets IV, ed. V. Mannings, A. Boss, \& S. Russel (Tucson: Univ. Arizona Press), 867 
Ridge, N. A., Wilson, T. L., Megeath, S. T., Allen, L. E., \& Myers, P. C. 2003, AJ, 126, 286

Rodriguez, L. F., Anglada, G., \& Curiel, S. 1999, ApJS, 125, 427

Sandell, G., \& Knee, L. B. G. 2001, ApJ, 546, L49

Snell, R. L., Loren, R. B., \& Plambeck, R. L. 1980, ApJ, 239, L17
Strom, S. E., Vrba, F. J., \& Strom, K. M. 1976, AJ, 81, 314

Warin, S., Castets, A., Langer, W. D., Wilson, R. W., \& Pagani, L. 1996, A\&A, 306, 935

Welch, Wm. J., Hartmann, L., Helfer, T., \& Briceño, C. 2000, ApJ, 540, 362

Yu, K. C., Billawala, Y., \& Bally, J. 1999, AJ, 118, 2940 